
\title{
$\angle S$ Research Square \\ Development and Validation of a Novel Sepsis Biomarker based on Amino acid Profiling
}

\section{Sunyoung Ahn}

Yonsei University College of Medicine

\section{Su Hwan Lee}

Yonsei University College of Medicine

Kyung Soo Chung

Yonsei University College of Medicine

Nam Su Ku

Yonsei University College of Medicine

Young-Min Hyun

Yonsei University College of Medicine

\section{Sail Chun}

University of Ulsan College of Medicine

\section{Moo Suk Park}

Yonsei University College of Medicine

\section{Sang-Guk Lee ( $\nabla$ comforter6@yuhs.ac)}

Yonsei University College of Medicine https://orcid.org/0000-0003-3862-3660

\section{Research}

Keywords: sepsis, biomarker, metabolomics, mass-spectrometry, amino acids, kynurenine, tryptophan, arginine, phenylalanine

Posted Date: December 1st, 2020

DOI: https://doi.org/10.21203/rs.3.rs-115088/v1

License: (c) (i) This work is licensed under a Creative Commons Attribution 4.0 International License. Read Full License 


\section{Abstract}

\section{Background}

Sepsis is a potentially fatal condition influenced by pathogens and host factors. However, the current sepsis biomarkers, such as white blood cell count, C-reactive protein, and procalcitonin levels, show unsatisfactory performance in terms of diagnostic sensitivity and specificity in clinical practice. Thus, we developed and validated a new sepsis biomarker using amino acid profiling.

\section{Methods}

We used two independent groups. The training and validation groups included 161 and 22 healthy controls, 123 and 50 patients with systemic inflammatory response syndrome, and 115 and 45 patients with sepsis, respectively. We measured and analyzed the serum amino acid levels using mass spectrometry to select candidate amino acids that could differentiate sepsis from other conditions. Then, several possible multivariate indexes were developed by generating a formula with different combinations of candidate amino acids. The formula showing the best performance was selected and validated further.

\section{Results}

Kynurenine, tryptophan, phenylalanine, arginine, aspartic acid, glutamic acid, and glutamine were selected as candidate amino acids. Ten possible formulae were generated, and that with the highest diagnostic performance was selected. In the validation group, the area under the receiving operating characteristic curve of the selected multivariate index (0.931) was similar to that of procalcitonin (0.945). Moreover, the generated multivariate index showed potential as a prognostic marker.

\section{Conclusions}

Amino acid composition in patients with sepsis differs significantly from that in healthy individuals and in patients with only inflammation. The newly developed multivariate index is expected to be feasible as a sepsis biomarker in clinical practice in the near future.

\section{Background}

Sepsis is a condition influenced by infectious pathogens and host factors and is characterized by aberrant or deregulated host response and organ dysfunction. Sepsis is often lethal, leading to death $20-50 \%$ of severely affected patients. It is the second leading cause of death among patients in non-coronary intensive care units (ICUs) and the 10th cause of overall deaths in the United States [1]. Care of patients with sepsis costs approximately $\$ 50,000$ per patient, consequently leading to an annual economic burden of nearly $\$ 17$ billion in the United States [2].

Both immune and endothelial dysfunction are thought to contribute to high mortality rate in sepsis [3]; however, the exact underlying mechanisms are yet to be elucidated. Consequently, currently used sepsis biomarkers, such as white blood cell (WBC) count, C-reactive protein (CRP), and procalcitonin (PCT), show 
unsatisfactory performance in terms of diagnostic sensitivity and specificity in clinical practice [4]. The widely accepted infection biomarker in the clinical setting is PCT. However, PCT remains controversial for differentiation of systemic inflammatory response syndrome (SIRS) and sepsis because it has been described to be elevated after major surgery, a common cause of SIRS without underlying infection [5-7]. Therefore, further investigation for discovering better sepsis markers is warranted.

Since sepsis has complex pathogenesis, a multi-marker strategy, such as metabolomics based on mass spectrometry, may be helpful for developing novel biomarkers. Biological studies on human metabolites and proteins using mass spectrometry technology are ongoing for over a decade $[4,8]$. Recently, several studies focusing on the investigation of plasma metabolomic profiles as predictive signatures of ICU mortality in adult patients with sepsis have been published $[4,9,10]$. They clearly highlight the widespread metabolic abnormalities in patients with sepsis and septic shock and the interplay of several different biochemical pathways.

Although there are several studies on metabolomics in sepsis [11-13], few of them have considered serum amino acids as candidate markers [13]. Moreover, to date, no previous study has developed sepsis biomarker as a multivariate index. Here, we aimed to develop and validate a sepsis biomarker as a multivariate index based on amino acid profiling, after determining serum amino acid levels using liquid chromatographytandem mass spectrometry (LC-MS/MS).

\section{Methods}

\section{Participant recruitment}

In this study, a training group was used to develop the sepsis multivariate biomarker and a validation group to validate the developed biomarker. Each group included three subgroups: healthy controls, patients with SIRS, and patients with sepsis.

In the training group, the healthy control subgroup comprised examinees that underwent a regular medical checkup at the Severance Checkup Center (Seoul, Republic of Korea) between July 2018 and January 2019. Residual sera from 161 individuals without abnormal laboratory findings or underlying diseases were collected, aliquoted, and frozen at $-80^{\circ} \mathrm{C}$ until analysis. The subgroup of patients with SIRS comprised patients who underwent selective orthopedic surgery at the Severance Hospital between August 2018 and May 2019 and did not have any other underlying disease or condition, including infection. Serum from 123 patients was collected postoperatively on the operation day. Lastly, 115 specimens from patients with sepsis were collected from a pre-existing cohort at the Severance Hospital, "Cohort Study of Patients with Sepsis in Intensive Care Units," between June 2018 and May 2019. The inclusion criteria for sepsis were [14]: a) suspected infection, b) satisfaction of more than two of these sub-criteria -respiratory rate $\geq 22 /$ min, altered mentation, or systolic blood pressure $\leq 100 \mathrm{mmHg}, \mathrm{c}$ ) sequential organ failure assessment score $\geq 2$ within $24 \mathrm{~h}$ of admission to ICU or emergency room (ER). Moreover, if vasopressors were required to maintain the mean arterial pressure $\geq 65 \mathrm{mmHg}$ and serum lactate level $>2 \mathrm{mmol} / \mathrm{L}$, the condition was diagnosed as septic shock. However, patients with neutropenia due to human immunodeficiency virus infection or cancer 
chemotherapy treatment and patients who underwent cardiopulmonary resuscitation within $24 \mathrm{~h}$ of ICU or ER admission were not recruited.

For the validation group, 22 healthy controls were recruited from the outpatient clinic at the Severance Hospital. Residual sera from those without abnormal laboratory findings or underlying diseases were collected. For the SIRS subgroup, sera from 50 patients who underwent selective orthopedic surgery at the Severance Hospital were collected between July and August 2018. Forty-five sepsis specimens from another pre-existing cohort at the Severance Hospital, "Cohort Study of Patients with Sepsis Admitted via ER," were collected between June 2018 and February 2019. The inclusion and exclusion criteria of this sepsis subgroup were the same as in the cohort study of patients with sepsis in ICU.

This research was approved by the institutional review board of the Severance Hospital (approval number: 42018-0387), Seoul, Republic of Korea. We obtained informed consent from all study participants. The clinical and laboratory data of the participants were obtained by reviewing electronic medical records. All participants were $>19$ years old.

\section{Assay instruments and reagents}

For the measurement of the 23 amino acids, the Zivak amino acids kit (Zivak Technologies, Istanbul, Turkey), a quantitative LC-MS/MS analysis kit specific for amino acids in biological fluids, was used. The 23 amino acids measured were: alpha-aminobutyric acid, alanine, arginine (ARG), asparagine, aspartic acid (ASP), citrulline, glutamic acid (GLU), glutamine (GLN), glycine, histidine, hydroxyproline, isoleucine, leucine, lysine, methionine (MET), ornithine, phenylalanine (PHE), proline, serine, threonine, tyrosine (TYR), valine, and tryptophan (TRP) (Additional file 1). The serum samples were processed in strict accordance with the manufacturer's instructions. Additional information on the method used for measuring amino acid concentrations is provided in Additional file 2.

The concentration of kynurenine (KYN) was determined using the same LC-MS/MS system (additional information in Additional file 3). Serum CRP concentrations were measured by immunoturbidimetric assay using Cobas c 702 (Roche, Basel, Switzerland). Serum PCT levels were measured by electrochemiluminescence immunoassay using Cobas e 601 (Roche).

\section{Selection of candidate amino acids}

After measuring serum amino acid concentrations, partial least squares-discriminant analysis (PLS-DA) was conducted to assess subgroup clustering and identify the amino acids responsible for distinction in the training group. Furthermore, the median concentrations of the amino acids were compared among the three subgroups. We also compared the amino acid concentrations between two subgroups: sepsis and nonsepsis (control plus SIRS). Comprehensively, we selected the candidate amino acids that played a major role in subgroup distinction in PLS-DA and showed significant differences in median concentrations among subgroups.

\section{Development of sepsis biomarker using multivariate analysis}


After candidate amino acids were selected, we performed logistic regression analysis using the training group to make several multivariate index formulae with diverse combinations of the selected candidate amino acids. To screen for the most efficient multivariate index formula, each formula was assessed based on its diagnostic performance, including sensitivity, specificity, accuracy, and area under the receiver operating characteristic (ROC) curve (AUC). Among them, one multivariate index formula showing the best performance was determined as the novel sepsis biomarker developed in this study.

\section{Validation of the developed sepsis biomarker}

The clinical diagnostic performance of the selected multivariate index was evaluated in the validation group. Its performance was also compared to that of pre-existing sepsis biomarkers such as WBC, CRP, and PCT. Moreover, the possibility of use as a sepsis prognostic biomarker was also assessed by comparing the difference in the selected multivariate index between the survivor and non-survivor subgroups.

\section{Statistical analysis}

The Mann-Whitney Utest and Kruskal-Wallis test were used for comparing differences in the medians of several independent groups. To confirm that the subgroups were well separated, PLS-DA was performed. It was also used to identify the amino acid that exerted a significant influence for clustering the subgroups. To formulate multivariate indexes from the candidate amino acids, we applied logistic regression analysis to generate multivariate indexes using R studio software "caret" package (version 1.1.453, MA, USA). We used Analyse-it (version 5.40.2, Analyse-it Software, Ltd., Leeds, UK), R studio, GraphPad Prism (version 6, GraphPad Software, CA, USA), and SPSS (version 18, IBM, NY, USA) for the statistical analyses. Differences were considered statistically significant for $p$-values $<0.05$.

\section{Results}

\section{Participant characteristics}

The training and validation groups included 161 and 22 healthy controls, 123 and 50 patients with SIRS, and 115 and 45 patients with sepsis, respectively. The detailed features of each group are outlined in Table 1. There were significant differences among the three subgroups in many baseline characteristics, except for sex and height, in the training group. These characteristics are considered to be a result of disease progression. 
Table 1

Baseline characteristics of the participants in the training and validation groups further divided into three subgroups according to septic status.

\begin{tabular}{|c|c|c|c|c|c|c|c|c|}
\hline & \multicolumn{4}{|c|}{ Training group } & \multicolumn{4}{|c|}{ Validation group } \\
\hline & $\begin{array}{l}\text { Normal } \\
(\mathrm{N}= \\
161)\end{array}$ & $\begin{array}{l}\text { SIRS } \\
(N= \\
123)\end{array}$ & $\begin{array}{l}\text { Sepsis } \\
(\mathrm{N}= \\
115)\end{array}$ & $\begin{array}{l}\text { p- } \\
\text { value }\end{array}$ & $\begin{array}{l}\text { Normal } \\
(\mathrm{N}=22)\end{array}$ & $\begin{array}{l}\text { SIRS } \\
(N=50)\end{array}$ & $\begin{array}{l}\text { Sepsis } \\
(\mathrm{N}=45)\end{array}$ & $\begin{array}{l}\mathrm{p}- \\
\text { value }\end{array}$ \\
\hline Gender, M (\%) & $\begin{array}{l}85 \\
(52.8)\end{array}$ & $\begin{array}{l}53 \\
(43.1)\end{array}$ & $\begin{array}{l}49 \\
(42.6)\end{array}$ & 0.149 & $3(13.6)$ & $\begin{array}{l}31 \\
(62.0)\end{array}$ & $\begin{array}{l}11 \\
(42.3)\end{array}$ & $\begin{array}{l}< \\
0.001\end{array}$ \\
\hline \multirow[t]{2}{*}{ Age (years) } & 46.00 & 58.67 & 69.40 & \multirow{2}{*}{$<.001$} & 58.00 & 64.18 & 72.48 & \multirow[t]{2}{*}{0.001} \\
\hline & $\begin{array}{l}{[37.00} \\
54.00]\end{array}$ & $\begin{array}{l}{[44.54,} \\
70.98]\end{array}$ & $\begin{array}{l}\text { [59.67, } \\
77.64]\end{array}$ & & $\begin{array}{l}{[55.75,} \\
60.00]\end{array}$ & $\begin{array}{l}{[50.33,} \\
72.95]\end{array}$ & $\begin{array}{l}\text { [59.33, } \\
78.13]\end{array}$ & \\
\hline \multirow[t]{2}{*}{ Height (cm) } & 163.10 & 166.00 & 163.50 & \multirow[t]{2}{*}{0.062} & 155.00 & 169.00 & 164.00 & \multirow{2}{*}{$<.001$} \\
\hline & $\begin{array}{l}{[158.30,} \\
170.00]\end{array}$ & $\begin{array}{l}{[159.70,} \\
173.00]\end{array}$ & $\begin{array}{l}{[157.25,} \\
170.00]\end{array}$ & & $\begin{array}{l}{[150.00,} \\
156.50]\end{array}$ & $\begin{array}{l}{[163.00} \\
172.07]\end{array}$ & $\begin{array}{l}{[158.25,} \\
175.00]\end{array}$ & \\
\hline \multirow[t]{2}{*}{ Weight (kg) } & 59.80 & 69.10 & 58.50 & \multirow{2}{*}{$<.001$} & 60.00 & 67.95 & 60.00 & \multirow{2}{*}{$<.001$} \\
\hline & $\begin{array}{l}{[54.10} \\
69.60]\end{array}$ & $\begin{array}{l}{[59.50} \\
76.60]\end{array}$ & $\begin{array}{l}{[49.85} \\
68.50]\end{array}$ & & $\begin{array}{l}{[55.00} \\
64.50]\end{array}$ & $\begin{array}{l}{[61.00} \\
71.70]\end{array}$ & $\begin{array}{l}{[48.00} \\
63.30]\end{array}$ & \\
\hline \multirow[t]{2}{*}{ BMI } & 23.05 & 24.77 & 21.77 & \multirow{2}{*}{$\begin{array}{l}< \\
0.001\end{array}$} & 24.39 & 24.06 & 20.17 & \multirow{2}{*}{$\begin{array}{l}<.001 \\
0.001\end{array}$} \\
\hline & $\begin{array}{l}{[20.85,} \\
25.09]\end{array}$ & $\begin{array}{l}{[21.88,} \\
27.29]\end{array}$ & $\begin{array}{l}{[19.65,} \\
25.61]\end{array}$ & & $\begin{array}{l}{[23.52,} \\
26.45]\end{array}$ & $\begin{array}{l}{[22.26,} \\
24.77]\end{array}$ & $\begin{array}{l}{[18.45,} \\
22.84]\end{array}$ & \\
\hline \multirow[t]{2}{*}{ Glucose (mg/dL) } & 91.00 & 106.00 & 136.00 & \multirow{2}{*}{$\begin{array}{l}< \\
0.001\end{array}$} & 24.39 & 109.50 & 116.00 & \multirow{2}{*}{$<.001$} \\
\hline & $\begin{array}{l}{[86.00} \\
99.00]\end{array}$ & $\begin{array}{l}{[94.50} \\
134.50]\end{array}$ & $\begin{array}{l}{[114.00,} \\
194.00]\end{array}$ & & $\begin{array}{l}{[23.52,} \\
26.45]\end{array}$ & $\begin{array}{l}{[94.00} \\
132.75]\end{array}$ & $\begin{array}{l}{[104.00,} \\
212.00]\end{array}$ & \\
\hline \multirow[t]{2}{*}{ Creatinine(mg/dL) } & 0.75 & 0.82 & 1.23 & \multirow{2}{*}{$\begin{array}{l}< \\
0.001\end{array}$} & 16.20 & 0.90 & 1.30 & \multirow{2}{*}{$\begin{array}{l}<.001 \\
0.001\end{array}$} \\
\hline & $\begin{array}{l}{[0.64,} \\
0.88]\end{array}$ & $\begin{array}{l}{[0.66,} \\
1.04]\end{array}$ & $\begin{array}{l}{[0.71} \\
2.75]\end{array}$ & & $\begin{array}{l}{[14.40,} \\
19.90]\end{array}$ & $\begin{array}{l}{[0.71,} \\
1.29]\end{array}$ & $\begin{array}{l}{[0.90} \\
1.54]\end{array}$ & \\
\hline \multirow{2}{*}{$\begin{array}{l}\text { Total protein } \\
(\mathrm{g} / \mathrm{dL})\end{array}$} & 7.30 & 6.40 & 5.10 & \multirow{2}{*}{$\begin{array}{l}< \\
0.001\end{array}$} & 4.30 & 6.20 & 5.35 & \multirow{2}{*}{$\begin{array}{l}< \\
0.001\end{array}$} \\
\hline & $\begin{array}{l}{[7.10} \\
7.60]\end{array}$ & $\begin{array}{l}{[5.95} \\
6.70]\end{array}$ & $\begin{array}{l}{[4.70} \\
5.95]\end{array}$ & & $\begin{array}{l}{[4.20} \\
4.50]\end{array}$ & $\begin{array}{l}{[5.80} \\
6.60]\end{array}$ & $\begin{array}{l}{[5.00} \\
6.00]\end{array}$ & \\
\hline \multirow[t]{2}{*}{ Albumin (g/dL) } & 4.50 & 3.70 & 2.50 & \multirow{2}{*}{$\begin{array}{l}< \\
0.001\end{array}$} & 4.32 & 3.50 & 3.00 & \multirow{2}{*}{$\begin{array}{l}<.001 \\
0.001\end{array}$} \\
\hline & $\begin{array}{l}{[4.40,} \\
4.68]\end{array}$ & $\begin{array}{l}{[3.30,} \\
4.00]\end{array}$ & $\begin{array}{l}{[2.20} \\
2.90]\end{array}$ & & $\begin{array}{l}{[3.40,} \\
4.58]\end{array}$ & $\begin{array}{l}{[3.10} \\
3.90]\end{array}$ & $\begin{array}{l}{[2.70,} \\
3.20]\end{array}$ & \\
\hline \multirow[t]{2}{*}{ AST (IU/L) } & 22.00 & 19.00 & 34.00 & \multirow{2}{*}{$\begin{array}{l}< \\
0.001\end{array}$} & 17.00 & 19.00 & 40.00 & \multirow{2}{*}{$\begin{array}{l}< \\
0.001\end{array}$} \\
\hline & $\begin{array}{l}{[18.00,} \\
26.00]\end{array}$ & $\begin{array}{l}{[15.00,} \\
24.00]\end{array}$ & $\begin{array}{l}{[22.00,} \\
62.00]\end{array}$ & & $\begin{array}{l}\text { [14.00, } \\
21.25]\end{array}$ & $\begin{array}{l}{[14.00,} \\
22.75]\end{array}$ & $\begin{array}{l}\text { [22.00, } \\
75.50]\end{array}$ & \\
\hline
\end{tabular}




\begin{tabular}{|c|c|c|c|c|c|c|c|c|}
\hline \multirow{3}{*}{$\operatorname{ALT}(\mathrm{IU} / \mathrm{L})$} & \multicolumn{4}{|c|}{ Training group } & \multicolumn{4}{|c|}{ Validation group } \\
\hline & 17.00 & 16.00 & 19.00 & 0.079 & 0.65 & 17.50 & 25.50 & $<$ \\
\hline & $\begin{array}{l}{[13.00} \\
26.00]\end{array}$ & $\begin{array}{l}{[11.00} \\
24.00]\end{array}$ & $\begin{array}{l}{[11.50} \\
34.00]\end{array}$ & & $\begin{array}{l}{[0.57} \\
0.83]\end{array}$ & $\begin{array}{l}{[10.25,} \\
25.00]\end{array}$ & $\begin{array}{l}{[14.25} \\
38.50]\end{array}$ & \\
\hline \multirow[t]{2}{*}{ Bilirubin $(\mathrm{mg} / \mathrm{dL})$} & 1.00 & 0.70 & 0.50 & \multirow{2}{*}{$<.001$} & 0.75 & 0.50 & 0.85 & 0.004 \\
\hline & $\begin{array}{l}{[0.80} \\
1.30]\end{array}$ & $\begin{array}{l}{[0.45} \\
0.90]\end{array}$ & $\begin{array}{l}{[0.30} \\
1.10]\end{array}$ & & $\begin{array}{l}{[0.60} \\
0.90]\end{array}$ & $\begin{array}{l}{[0.40,} \\
0.78]\end{array}$ & $\begin{array}{l}{[0.40} \\
1.32]\end{array}$ & \\
\hline \multirow[t]{2}{*}{$\mathrm{Hb}(\mathrm{g} / \mathrm{dL})$} & 13.60 & 12.20 & 9.80 & \multirow{2}{*}{$<.001$} & 12.60 & 11.70 & 10.25 & 0.021 \\
\hline & $\begin{array}{l}{[12.80} \\
14.50]\end{array}$ & $\begin{array}{l}{[10.60} \\
13.50]\end{array}$ & $\begin{array}{l}\text { [8.35, } \\
11.55]\end{array}$ & & $\begin{array}{l}\text { [12.45, } \\
13.05]\end{array}$ & $\begin{array}{l}{[9.72,} \\
13.17]\end{array}$ & $\begin{array}{l}{[8.75,} \\
12.00]\end{array}$ & \\
\hline \multirow[t]{2}{*}{ Hct (\%) } & 41.50 & 37.10 & 30.60 & \multirow{2}{*}{$<.001$} & 36.90 & 36.10 & 30.55 & 0.016 \\
\hline & $\begin{array}{l}{[39.40,} \\
44.10]\end{array}$ & $\begin{array}{l}{[33.10} \\
40.50]\end{array}$ & $\begin{array}{l}{[25.30} \\
35.50]\end{array}$ & & $\begin{array}{l}{[36.30} \\
37.85]\end{array}$ & $\begin{array}{l}{[29.12,} \\
39.72]\end{array}$ & $\begin{array}{l}{[27.07} \\
35.10]\end{array}$ & \\
\hline \multirow[t]{2}{*}{ Platelet $\left(10^{3} / \mathrm{uL}\right)$} & 244.50 & 237.00 & 179.00 & \multirow{2}{*}{$<.001$} & 247.00 & 238.50 & 196.00 & 0.005 \\
\hline & $\begin{array}{l}{[204.50,} \\
279.00]\end{array}$ & $\begin{array}{l}{[196.00} \\
300.00]\end{array}$ & $\begin{array}{l}{[79.50} \\
288.00]\end{array}$ & & $\begin{array}{l}{[224.00} \\
248.50]\end{array}$ & $\begin{array}{l}{[181.50,} \\
306.00]\end{array}$ & $\begin{array}{l}{[157.00} \\
218.00]\end{array}$ & \\
\hline \multirow[t]{2}{*}{ WBC $\left(10^{3} / \mathrm{uL}\right)$} & 5.16 & 7.65 & 10.91 & \multirow{2}{*}{$<.001$} & 6.28 & 7.90 & 10.28 & 0.011 \\
\hline & $\begin{array}{l}{[4.36} \\
5.81]\end{array}$ & $\begin{array}{l}{[6.05} \\
9.35]\end{array}$ & $\begin{array}{l}{[7.22,} \\
15.25]\end{array}$ & & $\begin{array}{l}{[5.43,} \\
7.69]\end{array}$ & $\begin{array}{l}{[5.73,} \\
10.09]\end{array}$ & $\begin{array}{l}{[5.25} \\
15.81]\end{array}$ & \\
\hline \multirow{2}{*}{$\begin{array}{l}\text { Procalcitonin } \\
(\mathrm{ng} / \mathrm{mL})\end{array}$} & \multirow{2}{*}{$\begin{array}{l}0.02 \\
{[0.02} \\
0.03]\end{array}$} & 0.04 & 1.99 & \multirow{2}{*}{$\begin{array}{l}<.001 \\
0.001\end{array}$} & 0.02 & 0.04 & 11.24 & \multirow{2}{*}{$\dot{0.001}$} \\
\hline & & $\begin{array}{l}{[0.02,} \\
0.09]\end{array}$ & $\begin{array}{l}{[0.52,} \\
11.24]\end{array}$ & & $\begin{array}{l}{[0.02} \\
0.06]\end{array}$ & $\begin{array}{l}{[0.02,} \\
0.33]\end{array}$ & $\begin{array}{l}{[1.17} \\
28.35]\end{array}$ & \\
\hline \multirow[t]{2}{*}{ CRP (mg/L) } & 0.56 & 7.09 & 128.90 & \multirow{2}{*}{$\begin{array}{l}< \\
0.001\end{array}$} & 1.00 & 9.25 & 106.95 & \multirow{2}{*}{$\begin{array}{l}<.001 \\
0.00\end{array}$} \\
\hline & $\begin{array}{l}{[0.33} \\
1.23]\end{array}$ & $\begin{array}{l}{[1.47} \\
42.88]\end{array}$ & $\begin{array}{l}{[47.90} \\
227.80]\end{array}$ & & $\begin{array}{l}{[0.85} \\
7.21]\end{array}$ & $\begin{array}{l}{[1.43,} \\
43.22]\end{array}$ & $\begin{array}{l}\text { [35.50, } \\
184.45]\end{array}$ & \\
\hline \multicolumn{9}{|c|}{$\begin{array}{l}\text { M: male, BMI: body mass index, AST: aspartate aminotransferase, ALT: alanine aminotransferase, Hb: } \\
\text { hemoglobin, Hct: hematocrit, WBC: white blood cell, CRP: C-reactive protein }\end{array}$} \\
\hline \multicolumn{9}{|c|}{ Data (except those for sex) are expressed as medians (interquartile ranges). } \\
\hline
\end{tabular}

\section{Selected candidate amino acids}

Based on the score plot of PLS-DA (Fig. 1a), we confirmed that the three subgroups of the training group were clearly distinguishable and formed distinct clusters. This result suggests that amino acid profiling could be applied successfully for distinguishing those with and without sepsis. The loading plot of PLS-DA indicated that the amino acids most responsible for the distinction of the sepsis subgroup were: KYN, TRP, ARG, PHE, and ASP (Fig. 1b). In the importance plot of PLS-DA, the five aforementioned amino acids and GLU are located at the top of the plot (Fig. 1c). 
To identify the candidate amino acids, we also performed a comparison analysis of amino acid concentrations in the training group using the Mann-Whitney Uand Kruskal-Wallis tests (Fig. 2, Additional file 1). We found that the concentrations of KYN, TRP, PHE, ARG, ASP, and GLU, which were important in the previous PLS-DA, were significantly different $(p$-value $<0.05)$ between patients with and without sepsis (Fig. 2), as well as among healthy controls and patients with SIRS and sepsis (Additional file 1).

From the PLS-DA and the Mann-Whitney $U$ and Kruskal-Wallis tests, the candidate amino acids selected were $K Y N$, TRP, PHE, ARG, ASP, and GLU. We also added GLN as candidate because GLN and GLU can be metabolized to each other through glutamine synthase and glutaminase. Moreover, immune system-related cells, including lymphocytes and macrophages, have higher GLN requirements during inflammatory states such as sepsis; therefore, serum GLN level decreases $[15,16]$. We also calculated KYN/TRP ratio (KT ratio), as these two amino acids turn into opposite reflections and are related to each other in the metabolic pathway $[17,18]$. The GLN and KT ratio also showed significant difference between patients with and without sepsis (Fig. 2, Additional file 5) [7, 17-20]. Finally, we decided to use all eight abovementioned indicators as candidate amino acids (and the ratio) for developing the multivariate biomarker for sepsis.

\section{Development of new multivariate index as a sepsis biomarker}

With the selected candidate amino acids (and the ratio), we developed 10 multivariate index formulae by logistic regression analysis (Table 2). The performance of each generated formula was evaluated based on the corresponding AUC, sensitivity, specificity, and accuracy using data from the training group. Finally, the third multivariate index formula comprising KT ratio, ARG, and PHE was selected based on its performance in discriminating sepsis (Table 2). Although the third multivariate index formula had fewer variables than the second, which additionally included GLU and GLN, the performance of the third multivariate index formula was better than that of the second. 
Table 2

Results of multivariate index formulae determined by logistic regression for the training group. All the formulae and the corresponding AUC, cut-off, sensitivity, specificity, and accuracy are outlined. Formula No.

3 was selected as the novel multivariate index for sepsis biomarker in this study.

\begin{tabular}{|c|c|c|c|c|c|c|}
\hline $\begin{array}{l}\text { Formula } \\
\text { No. }\end{array}$ & $\begin{array}{l}\text { Factors and Generated } \\
\text { Formula }\end{array}$ & AUC & cut-off & Sensitivity & Specificity & Accuracy \\
\hline \multirow[t]{2}{*}{1} & $\begin{array}{l}\text { KT ratio, ARG, PHE, GLU, GLN, } \\
\text { ASP }\end{array}$ & \multirow[t]{2}{*}{0.9870} & \multirow[t]{2}{*}{-1.0460} & 0.9331 & 0.9478 & 0.9373 \\
\hline & $\begin{array}{l}\text { KT ratio } \times 25.8002-A R G \times \\
0.0519+\mathrm{PHE} \times 0.0570-\mathrm{GLU} \times \\
0.0218-\mathrm{GLN} \times 0.0034+\mathrm{ASP} \times \\
0.0275-3.5413\end{array}$ & & & $\begin{array}{l}{[0.8958,} \\
0.9581]\end{array}$ & $\begin{array}{l}{[0.8852,} \\
0.9786]\end{array}$ & $\begin{array}{l}{[0.9089,} \\
0.9590]\end{array}$ \\
\hline \multirow[t]{2}{*}{2} & KT ratio, ARG, PHE, GLU, GLN & \multirow[t]{2}{*}{0.9880} & \multirow[t]{2}{*}{-1.2880} & 0.9366 & 0.9652 & 0.9449 \\
\hline & $\begin{array}{l}\text { KT ratio } \times 26.5502-A R G \times \\
0.0492+\mathrm{PHE} \times 0.0628+\mathrm{GLU} \times \\
0.0013-\mathrm{GLN} \times 0.0037-3.8763\end{array}$ & & & $\begin{array}{l}{[0.8999} \\
0.9609]\end{array}$ & $\begin{array}{l}{[0.9080} \\
0.9888]\end{array}$ & $\begin{array}{l}{[0.9177,} \\
0.9651]\end{array}$ \\
\hline \multirow[t]{2}{*}{3} & $\mathrm{KT}$ ratio, $\mathrm{ARG}, \mathrm{PHE}$ & \multirow[t]{2}{*}{0.9870} & \multirow[t]{2}{*}{-1.1280} & 0.9401 & 0.9652 & 0.9474 \\
\hline & $\begin{array}{l}\mathrm{KT} \text { ratio } \times 27.7705-\mathrm{ARG} \times \\
0.0515+\mathrm{PHE} \times 0.0643-5.4721\end{array}$ & & & $\begin{array}{l}{[0.9041} \\
0.9637]\end{array}$ & $\begin{array}{l}{[0.9081} \\
0.9888]\end{array}$ & $\begin{array}{l}{[0.9207} \\
0.9671]\end{array}$ \\
\hline \multirow[t]{2}{*}{4} & KYN, TRP, ARG, PHE & \multirow[t]{2}{*}{0.9870} & \multirow[t]{2}{*}{-1.1390} & 0.9266 & 0.9652 & 0.9398 \\
\hline & $\begin{array}{l}\mathrm{KYN} \times 0.3925-\mathrm{TRP} \times 0.0937- \\
\text { ARG } \times 0.0516+\mathrm{PHE} \times 0.0688- \\
1.0310\end{array}$ & & & $\begin{array}{l}{[0.8917} \\
0.9553]\end{array}$ & $\begin{array}{l}{[0.9081} \\
0.9887]\end{array}$ & $\begin{array}{l}0.9118 \\
0.9611]\end{array}$ \\
\hline \multirow[t]{2}{*}{5} & KT ratio, ARG, $\mathrm{PHE}, \mathrm{ASP}$ & \multirow[t]{2}{*}{0.9670} & \multirow[t]{2}{*}{-1.2370} & 0.9366 & 0.9565 & 0.9424 \\
\hline & $\begin{array}{l}\text { KT ratio } \times 26.1522-A R G \times \\
0.0541+\mathrm{PHE} \times 0.0547+\mathrm{ASP} \times \\
0.0312-5.0448\end{array}$ & & & $\begin{array}{l}{[0.8999} \\
0.9609]\end{array}$ & $\begin{array}{l}{[0.9565} \\
0.9839]\end{array}$ & $\begin{array}{l}{[0.9148} \\
0.9631]\end{array}$ \\
\hline \multirow[t]{2}{*}{6} & KT ratio, $\mathrm{PHE}, \mathrm{GLU}, \mathrm{GLN}$ & \multirow[t]{2}{*}{0.9760} & \multirow[t]{2}{*}{-1.1590} & 0.9437 & 0.9391 & 0.9424 \\
\hline & $\begin{array}{l}\text { KT ratio } \times 28.4968+\text { PHE } \times \\
0.0399+\text { GLU } \times 0.0042-G L N \times \\
0.0053-5.7122\end{array}$ & & & $\begin{array}{l}{[0.9083} \\
0.9663]\end{array}$ & $\begin{array}{l}{[0.8742,} \\
0.9731]\end{array}$ & $\begin{array}{l}{[0.9148} \\
0.9631]\end{array}$ \\
\hline \multirow[t]{2}{*}{7} & KT ratio, ARG, GLU, GLN & \multirow[t]{2}{*}{0.9600} & \multirow[t]{2}{*}{-1.0440} & 0.9225 & 0.9217 & 0.9223 \\
\hline & $\begin{array}{l}\text { KT ratio } \times 33.1247-A R G \times \\
0.0229+\mathrm{GLU} \times 0.0187-\mathrm{GLN} \times \\
0.0002-3.4614\end{array}$ & & & $\begin{array}{l}{[0.8835} \\
0.9497]\end{array}$ & $\begin{array}{l}{[0.8526} \\
0.9613]\end{array}$ & $\begin{array}{l}{[0.8915} \\
0.946]\end{array}$ \\
\hline \multirow[t]{2}{*}{8} & KT ratio, ARG & \multirow[t]{2}{*}{0.9430} & \multirow[t]{2}{*}{-0.9170} & 0.9331 & 0.8783 & 0.9173 \\
\hline & $\begin{array}{l}\text { KT ratio } \times 36.2083-A R G \times \\
0.0230-1.4355\end{array}$ & & & $\begin{array}{l}{[0.8958,} \\
0.9581]\end{array}$ & $\begin{array}{l}{[0.8009} \\
0.9294]\end{array}$ & $\begin{array}{l}{[0.8858,} \\
0.9424]\end{array}$ \\
\hline \multirow[t]{2}{*}{9} & $\mathrm{KT}$ ratio, $\mathrm{PHE}$ & \multirow[t]{2}{*}{0.9740} & \multirow[t]{2}{*}{-1.2870} & 0.9261 & 0.9391 & 0.9298 \\
\hline & $\begin{array}{l}\text { KT ratio } \times 30.1206+\text { PHE } \times \\
0.0416-8.0384\end{array}$ & & & $\begin{array}{l}{[0.8876,} \\
0.9525]\end{array}$ & $\begin{array}{l}{[0.8742,} \\
0.9731]\end{array}$ & $\begin{array}{l}{[0.9002,} \\
0.9529]\end{array}$ \\
\hline
\end{tabular}




\begin{tabular}{|llccccc|}
\hline $\begin{array}{l}\text { Formula } \\
\text { No. }\end{array}$ & $\begin{array}{l}\text { Factors and Generated } \\
\text { Formula }\end{array}$ & AUC & cut-off & Sensitivity & Specificity & Accuracy \\
\hline 10 & KT ratio & 0.9430 & -2.0510 & 0.8732 & 0.9304 & 0.8897 \\
& KT ratio $\times 38.7780-3.8869$ & & & {$[0.8275$,} & {$[0.8633$,} & {$[0.8548$,} \\
& & & $0.9085]$ & $0.9673]$ & $0.9187]$ \\
\hline
\end{tabular}

\section{Validation of the developed multivariate index as a sepsis diagnostic biomarker}

After selecting the final multivariate index for sepsis diagnosis biomarker, we validated its diagnostic performance and its potential as a prognostic biomarker by comparing the levels of the new multivariate index and pre-existing sepsis biomarkers (WBC, CRP, and PCT) among the three subgroups of the validation group using the Kruskal-Wallis test (Fig. 3). The multivariate index indicated good performance as it could discriminate between normal and SIRS, SIRS and sepsis, and normal and sepsis. PCT and CRP could discriminate sepsis from non-sepsis relatively well; however, the discrimination performance between normal status and SIRS was poor. WBC hardly discriminated sepsis from normal, indicating poor diagnostic performance. However, the Mann-Whitney $U$ test between two subgroups (non-sepsis and sepsis) showed significant differences for all markers (Additional file 6).

The ability of the new multivariate index, WBC, CRP, PCT, and KT ratio to discriminate between sepsis and non-sepsis in the validation group was determined using ROC curves (Table 3, Additional file 7). The AUC values were 0.93 for the multivariate index (95\% confidence interval [Cl], $0.89-0.97), 0.95$ for PCT $(95 \% \mathrm{Cl}$, $0.90-0.99), 0.80$ for CRP ( $95 \% \mathrm{Cl}, 0.71-0.89), 0.64$ for WBC ( $95 \% \mathrm{Cl}, 0.51-0.76)$, and 0.84 for KT ratio (95\% $\mathrm{Cl}, 0.76-0.92)$. Only the multivariate index and PCT showed AUCs $>0.9$, which is a significantly high score in clinical practice. Although PCT showed higher AUC than that of the multivariate index, the difference was not statistically significant (De Long's test, $p$-value $=0.9903$ ). This result indicates that the multivariate index or PCT are better suited as diagnostic biomarkers for sepsis when compared with WBC, CRP, or KT ratio.

Furthermore, the multivariate index showed statistically significant difference between survivors and nonsurvivors in the validation group (Mann-Whitney $U$ test, $p$-value $=0.019)$ (Additional file 8). However, PCT concentration did not differ between survivors and non-survivors. Therefore, the newly developed multivariate index could be used as a prognostic biomarker in early sepsis and to differentially diagnose sepsis. 
Table 3

The AUCs, $95 \%$ confidence intervals of AUCs, and SEs of sepsis biomarkers including the developed multivariate index from the validation group. The Cls for procalcitonin and the developed multivariate index overlap, which indicates that there may not be significant difference between the AUCs of the two biomarkers.

\begin{tabular}{|llll|}
\hline & AUC & $95 \%$ Cl & SE \\
\hline Multivariate index & 0.931 & 0.887 to 0.974 & 0.0222 \\
\hline Procalcitonin & 0.945 & 0.904 to 0.986 & 0.0209 \\
\hline KT Ratio & 0.835 & 0.756 to 0.915 & 0.0403 \\
\hline CRP & 0.796 & 0.706 to 0.885 & 0.0457 \\
\hline WBC & 0.635 & 0.514 to 0.755 & 0.0614 \\
\hline $\begin{array}{l}\text { AUC: area under the receiving operating characteristic curve, Cl: confidence interval, SE: standard error, } \\
\text { KT ratio: kynurenine/tryptophan ratio, CRP: C-reactive protein, WBC: white blood cell }\end{array}$ \\
\hline
\end{tabular}

\section{Discussion}

Herein, we found that amino acid metabolism in patients with sepsis is different from that in healthy individuals and patients with SIRS; indeed, the serum concentrations of some amino acids were significantly increased or decreased in patients with sepsis. We utilized these amino acids to develop a sepsis diagnostic biomarker in the form of multivariate index and validated its performance in the diagnosis of sepsis. The developed multivariate biomarker showed excellent performance in sepsis diagnosis with an AUC of 0.931 and exhibited a potential as sepsis prognostic biomarker.

In clinical practice, patients with sepsis typically exhibit significant heterogeneity regarding variables such as age, presence of underlying or secondary disease, state of the immune system, and infection severity. Moreover, the term "sepsis" covers a wide variety of pathophysiological processes that occur following infection [21]. Such features of heterogeneity increase the challenges of diagnosing sepsis timely and choosing the proper treatment methods for each patient. Therefore, a metabolomic approach would be preferred in treatment decision-making and prognosis of sepsis. Several researches have studied metabolomic derangements in sepsis [9, 22, 23]. Ferrario et al. [22] showed that low unsaturated long-chain phosphatidylcholine and lysophosphatidylcholine species were associated with long-term survival (90-day)

together with circulating KYN in patients with septic shock. Rogers et al. [9] reported that due to metabolomic derangements, some metabolites, including KYN, were closely associated with 28-day mortality in critically ill patients. Furthermore, Cambiaghi et al. [12] reported that certain metabolites including KYN were associated with treatment responsiveness in the acute phase of septic shock. Although there are several previous studies on sepsis metabolism, this is the first attempt to develop sepsis diagnostic biomarker as a multivariate index using serum amino acid profiling.

In the selection of candidate amino acids for developing the multivariate index, we considered the results of PLS-DA and comparison analysis of amino acid concentrations among subgroups, as well as data from the 
literature. Based on recent studies, all amino acids (TRP, ARG, and PHE) and the metabolite (KYN) included in the new multivariate index are closely associated with metabolic processes in patients with sepsis. The catabolic conversion of TRP to KYN occurs in sepsis by indoleamine-2,3-dioxygenase (IDO) [17, 19, 20, 24]. IDO is induced by interferon-gamma, toll-like receptors, and bacterial DNA, which are molecules related to infection and inflammation $[18,24,25]$. Therefore, KYN, TRP, and KT ratio are widely evaluated as indicators for sepsis, and recent findings have clearly revealed that increased KT ratio due to elevated IDO expression is a hallmark of bacterial infections such as in sepsis $[25,26]$. ARG is an important initiator of immune response $[27,28]$. Patients with sepsis present reduction in ARG synthesis in the kidney and increase in the synthesis of acute phase proteins, which contain ARG. Consequentially, ARG catabolism overrides anabolism in sepsis, and the serum levels of ARG decrease [29,30]. PHE, which is a necessary precursor in the synthesis of catecholamines and thyroid hormone, is converted to TYR in the liver by phenylalanine hydroxylase (PAH), which is a sensitive hepatic enzyme [31]. Recently, a study revealed that increased PHE level in sepsis is indicative of insufficient conversion to TYR by PAH owing to damage caused to PAH cofactor, 5,6,7,8-tetrahydrobiopterin, by oxidative stress resulting from immune activation, such as sepsis [32]. Therefore, we could confirm that appropriate candidate amino acids, pathophysiologically related to sepsis, were selected to develop the multivariate index.

For the diagnostic performance of KT ratio, Zeden et al. [19] reported that the diagnostic AUC of KT ratio was $0.788(95 \% \mathrm{Cl}, 0.644-0.912)$ in sepsis. However, in our study, the AUC of KT ratio alone was 0.835 and that of the multivariate index, which included PHE and ARG in addition to KT ratio, was $0.931(95 \% \mathrm{Cl}, 0.887-$ 0.974). Adding these two amino acids to the novel multivariate index showed better performance than that observed using KT ratio alone; however, further addition of amino acids such as ASP, GLU, and GLN did not improve the index clinical performance.

There are some limitations in our study. First, most of the pathogens infecting the patients with sepsis in this study were bacteria; there were few cases of fungal or viral infections. Since it is well known that serum PCT levels are significantly lower in patients with viral or fungal infections than in patients with bacterial infections [33,34], these conditions could not be diagnosed appropriately using PCT. Moreover, patients with neutropenia were excluded from the study; however, PCT is produced by WBCs to some extent and, thus, the diagnostic performance of PCT in neutropenic status is expected to decrease [35]. In our study, the newly developed index and PCT showed the same excellent performance in sepsis diagnosis, and there was no significant difference between the two indicators. Therefore, to confirm whether the multivariate index has more strength in diagnosis of sepsis compared to PCT, a comparative study using patients with neutropenia or fungal infections is needed. Second, most of the patients with sepsis in the validation group had severe septic shock, which might have influenced the results of early sepsis biomarker evaluation. Septic shock is the most severe state of sepsis; therefore, more extensive biomarker changes may also appear. Previous studies using PCT have reported that PCT concentration was higher in septic shock than in sepsis cases, reflecting its severity [36, 37]. In our study, the AUC of both PCT and multivariate index was higher than 0.9 in the validation group. This value is relatively high when compared to those of other studies; Tang et al. [7] reported in their meta-analysis that the diagnostic performance of PCT, with an AUC of $0.78(95 \% \mathrm{Cl}, 0.73-$ 0.83), was not satisfactory for diagnosing sepsis. Tsalik et al. [37] also reported similar performance (AUC 0.78) of PCT predicting septicemia, which was relatively high compared to CRP and interleukin-6. Samraj et 
al. [38] reported better clinical performance of PCT with an AUC of $0.84(95 \% \mathrm{Cl}, 0.75-0.9)$ for discriminating sepsis in children with cardiopulmonary bypass, but it was still lower than that in our study. Since most of the patients with sepsis in our validation group had severe shock, the diagnostic performance might have been overestimated compared to that in other studies. Therefore, further research is needed to confirm whether the multivariate index also shows a high diagnostic performance of an AUC of 0.9 or higher even at the early stage of sepsis unlike PCT. Finally, the novel multivariate index in our study is based on amino acid concentration measured by LC-MS/MS. However, LC-MS/MS is not widely distributed in front-line laboratories due to high cost, need of well-trained personnel, and low test throughput when compared to previously used automated chemistry analyzers. However, recently, matrix-assisted laser desorption ionization-time-of-flight mass spectrometer of small-size or for point-of-care testing has been introduced, and this type of point-of-care assay can be used instead of LC-MS/MS for amino acid concentration measurements with an easier operation method and short turnaround time, which improves the applicability of the test [39].

To our knowledge, this is the first study that utilized amino acid profiling for sepsis marker development using multivariate index. Moreover, we validated this multivariate marker for sepsis diagnosis in an independent patient group. It also included patients with SIRS, which is a condition barely distinguishable from sepsis when using pre-existing sepsis biomarkers. Furthermore, this study might improve the understanding of clinicians and researchers on the applicability of metabolomics in clinical medicine and the utility of multivariate index analysis as a tool for developing multivariate biomarkers showing good performance.

Concluding, we generated a simple and of high-performance multivariate sepsis biomarker, highly specific for sepsis diagnosis using only four serum amino acid concentrations. Moreover, it showed potential as sepsis prognostic biomarker. Our study demonstrated that serum amino acid profiling in the diagnosis of sepsis could yield promising results because it depicts the individual characteristic metabolic state, which would enable and help clinicians to use tailored medicine, ensure early diagnosis and timely adequate treatment, and precisely predict prognosis.

\section{Abbreviations}

ARG: arginine, ASP: aspartic acid, AUC: area under the receiver operating characteristic curve, Cl: confidence interval, CRP: C-reactive protein, ER: emergency room, GLN: glutamine, GLU: glutamic acid, ICU: intensive care unit, IDO: indoleamine-2,3-dioxygenase, KT ratio: KYN/TRP ratio, KYN: kynurenine, LC-MS/MS: liquid chromatography-tandem mass-spectrometry, MET: methionine, PAH: phenylalanine hydroxylase, PCT: procalcitonin, PHE: phenylalanine, PLS-DA: partial least squares-discriminant analysis, ROC: receiver operating characteristic, SIRS: systemic inflammatory response syndrome, TRP: tryptophan, TYR: tyrosine, WBC: white blood cell

\section{Declarations}

\section{Ethics approval and consent to participate}


This research was approved by the institutional review board of the Severance Hospital (approval number: 42018-0387), Seoul, Republic of Korea. We obtained informed consent from all study participants.

\section{Consent for publication}

Not applicable.

\section{Availability of data and materials}

The datasets used for the analysis in the current study are available from the corresponding author on reasonable request.

\section{Competing interests}

The authors declare that they have no competing interests.

\section{Funding}

This study was supported by a Grant from the Ministry of Health \& Welfare, Republic of Korea [Grant number: HI14C1324]. It was also supported by a faculty research grant of Yonsei University College of Medicine [6-2020-0073].

\section{Authors' contributions}

All authors confirmed they have contributed to the content of this paper and have fulfilled their role faithfully. S. Ahn: statistical analysis, interpretation of data, drafting and revising the article; S. H. Lee: recruiting the participants, drafting and revising the article; K. S. Chung: recruiting the participants; N. S. Ku: provision of study material and patients; Y. -M. Hyun: statistical analysis; S. Chun: interpretation of data; M. S. Park: design the study and revising the article; and S. - G. Lee: design the study, interpretation of data, drafting and revising the article

\section{Acknowledgements}

We would like to thank the study participants for their cooperation and support and for providing their consent regarding the publication of this manuscript. We also thank the medical staff for the collection of blood samples.

\section{References}


1. Pittet D, Rangel-Frausto S, Li N, Tarara D, Costigan M, Rempe L, Jebson P, Wenzel RP: Systemic inflammatory response syndrome, sepsis, severe sepsis and septic shock: Incidence, morbidities and outcomes in surgical ICU patients. Intensive Care Medicine 1995, 21(4):302-309.

2. Martin GS, Mannino DM, Eaton S, Moss M: The epidemiology of sepsis in the United States from 1979 through 2000. N Engl J Med 2003, 348(16):1546-1554.

3. Hotchkiss RS, Karl IE: The pathophysiology and treatment of sepsis. N Engl J Med 2003, 348(2):138150.

4. Schmerler D, Neugebauer S, Ludewig K, Bremer-Streck S, Brunkhorst FM, Kiehntopf M: Targeted metabolomics for discrimination of systemic inflammatory disorders in critically ill patients. $J$ Lipid Res 2012, 53(7):1369-1375.

5. Meisner M, Tschaikowsky K, Hutzler A, Schick C, Schmidt J: Postoperative plasma concentrations of procalcitonin after different types of surgery. Critical Care 1998, 2(Suppl 1):P040-P040.

6. Mimoz O, Benoist JF, Edouard AR, Assicot M, Bohuon C, Samii K: Procalcitonin and C-reactive protein during the early posttraumatic systemic inflammatory response syndrome. Intensive Care Med 1998, 24(2):185-188.

7. Tang BM, Eslick GD, Craig JC, McLean AS: Accuracy of procalcitonin for sepsis diagnosis in critically ill patients: systematic review and meta-analysis. Lancet Infect Dis 2007, 7(3):210-217.

8. Crutchfield CA, Thomas SN, Sokoll LJ, Chan DW: Advances in mass spectrometry-based clinical biomarker discovery. Clin Proteomics 2016, 13:1.

9. Rogers AJ, McGeachie M, Baron RM, Gazourian L, Haspel JA, Nakahira K, Fredenburgh LE, Hunninghake GM, Raby BA, Matthay MA et al: Metabolomic derangements are associated with mortality in critically ill adult patients. PLoS One 2014, 9(1):e87538.

10. Mickiewicz B, Duggan GE, Winston BW, Doig C, Kubes P, Vogel HJ: Metabolic profiling of serum samples by $1 \mathrm{H}$ nuclear magnetic resonance spectroscopy as a potential diagnostic approach for septic shock. Crit Care Med 2014, 42(5):1140-1149.

11. Wang J, Sun Y, Teng S, Li K: Prediction of sepsis mortality using metabolite biomarkers in the blood: a meta-analysis of death-related pathways and prospective validation. BMC Medicine 2020, 18(1):83.

12. Cambiaghi A, Pinto BB, Brunelli L, Falcetta F, Aletti F, Bendjelid K, Pastorelli R, Ferrario M: Characterization of a metabolomic profile associated with responsiveness to therapy in the acute phase of septic shock. Scientific Reports 2017, 7(1):9748.

13. W Chen CS, L Chen, Q Kou: Metabonomics analysis of sepsis and non-infected SIRS patients based on mass spectrometry. Int J Clin Exp Med 2019, 2019;12:5023-5032.

14. Singer M, Deutschman CS, Seymour CW, Shankar-Hari M, Annane D, Bauer M, Bellomo R, Bernard GR, Chiche JD, Coopersmith CM et al: The Third International Consensus Definitions for Sepsis and Septic Shock (Sepsis-3). Jama 2016, 315(8):801-810.

15. de Oliveira DC, da Silva Lima F, Sartori T, Santos ACA, Rogero MM, Fock RA: Glutamine metabolism and its effects on immune response: molecular mechanism and gene expression. Nutrire 2016, 41(1):14.

16. Karinch AM, Pan M, Lin CM, Strange R, Souba WW: Glutamine metabolism in sepsis and infection. $J$ Nutr 2001, 131(9 Suppl):2535S-2538S; discussion 2550S-2531S. 
17. Logters TT, Laryea MD, Altrichter J, Sokolowski J, Cinatl J, Reipen J, Linhart W, Windolf J, Scholz M, Wild $\mathrm{M}$ : Increased plasma kynurenine values and kynurenine-tryptophan ratios after major trauma are early indicators for the development of sepsis. Shock 2009, 32(1):29-34.

18. Badawy AAB, Guillemin G: The Plasma [Kynurenine]/[Tryptophan] Ratio and Indoleamine 2,3Dioxygenase: Time for Appraisal. Int J Tryptophan Res 2019, 12:11786469198689781178646919868978.

19. Zeden JP, Fusch G, Holtfreter B, Schefold JC, Reinke P, Domanska G, Haas JP, Gruendling M, Westerholt A, Schuett C: Excessive tryptophan catabolism along the kynurenine pathway precedes ongoing sepsis in critically ill patients. Anaesth Intensive Care 2010, 38(2):307-316.

20. Changsirivathanathamrong D, Wang Y, Rajbhandari D, Maghzal GJ, Mak WM, Woolfe C, Duflou J, Gebski V, dos Remedios CG, Celermajer DS et al: Tryptophan metabolism to kynurenine is a potential novel contributor to hypotension in human sepsis. Crit Care Med 2011, 39(12):2678-2683.

21. Jensen JU, Bouadma L: Why biomarkers failed in sepsis. Intensive Care Med 2016, 42(12):2049-2051.

22. Ferrario M, Cambiaghi A, Brunelli L, Giordano S, Caironi P, Guatteri L, Raimondi F, Gattinoni L, Latini R, Masson $S$ et al: Mortality prediction in patients with severe septic shock: a pilot study using a target metabolomics approach. Sci Rep 2016, 6:20391.

23. Mickiewicz B, Tam P, Jenne CN, Leger C, Wong J, Winston BW, Doig C, Kubes P, Vogel HJ, for the Alberta Sepsis N: Integration of metabolic and inflammatory mediator profiles as a potential prognostic approach for septic shock in the intensive care unit. Critical Care 2015, 19(1):11.

24. Darcy CJ, Davis JS, Woodberry T, McNeil YR, Stephens DP, Yeo TW, Anstey NM: An observational cohort study of the kynurenine to tryptophan ratio in sepsis: association with impaired immune and microvascular function. PLoS One 2011, 6(6):e21185.

25. Schmidt SV, Schultze JL: New Insights into IDO Biology in Bacterial and Viral Infections. Frontiers in Immunology 2014, 5:384.

26. Suzuki Y, Suda T, Yokomura K, Suzuki M, Fujie M, Furuhashi K, Hahimoto D, Enomto N, Fujisawa T, Nakamura $Y$ et al: Serum activity of indoleamine 2,3-dioxygenase predicts prognosis of communityacquired pneumonia. J Infect 2011, 63(3):215-222.

27. Wu G, Morris SM, Jr.: Arginine metabolism: nitric oxide and beyond. Biochem J 1998, 336 (Pt 1)(Pt 1):117.

28. Wijnands KAP, Castermans TMR, Hommen MPJ, Meesters DM, Poeze M: Arginine and citrulline and the immune response in sepsis. Nutrients 2015, 7(3):1426-1463.

29. Luiking YC, Poeze M, Ramsay G, Deutz NE: The role of arginine in infection and sepsis. JPEN J Parenter Enteral Nutr 2005, 29(1 Suppl):S70-74.

30. O'Dwyer MJ, Dempsey F, Crowley V, Kelleher DP, McManus R, Ryan T: Septic shock is correlated with asymmetrical dimethyl arginine levels, which may be influenced by a polymorphism in the dimethylarginine dimethylaminohydrolase II gene: a prospective observational study. Crit Care 2006, 10(5):R139-R139.

31. Herndon DN, Wilmore DW, Mason AD, Jr., Pruitt BA, Jr.: Abnormalities of Phenylalanine and Tyrosine Kinetics: Significance in Septic and Nonseptic Burned Patients. Archives of Surgery 1978, 113(2):133- 
135.

32. Ploder M, Neurauter G, Spittler A, Schroecksnadel K, Roth E, Fuchs D: Serum phenylalanine in patients post trauma and with sepsis correlate to neopterin concentrations. Amino Acids 2008, 35(2):303-307.

33. Dornbusch HJ, Strenger V, Kerbl R, Lackner H, Schwinger W, Sovinz P, Urban C: Procalcitonin-a marker of invasive fungal infection? Support Care Cancer 2005, 13(5):343-346.

34. Assicot M, Gendrel D, Carsin H, Raymond J, Guilbaud J, Bohuon C: High serum procalcitonin concentrations in patients with sepsis and infection. Lancet 1993, 341(8844):515-518.

35. Oberhoffer M, Stonans I, Russwurm S, Stonane E, Vogelsang H, Junker U, Jäger L, Reinhart K: Procalcitonin expression in human peripheral blood mononuclear cells and its modulation by lipopolysaccharides and sepsis-related cytokines in vitro. J Lab Clin Med 1999, 134(1):49-55.

36. Bozza FA, Bozza PT, Castro Faria Neto HC: Beyond sepsis pathophysiology with cytokines: what is their value as biomarkers for disease severity? Memórias do Instituto Oswaldo Cruz 2005, 100:217-221.

37. Tsalik EL, Jaggers LB, Glickman SW, Langley RJ, van Velkinburgh JC, Park LP, Fowler VG, Cairns CB, Kingsmore SF, Woods CW: Discriminative value of inflammatory biomarkers for suspected sepsis. $J$ Emerg Med 2012, 43(1):97-106.

38. Samraj RS, Zingarelli B, Wong HR: Role of biomarkers in sepsis care. Shock 2013, 40(5):358-365.

39. Gogichaeva NV, Alterman MA: Amino Acid Analysis by Means of MALDI TOF Mass Spectrometry or MALDI TOF/TOF Tandem Mass Spectrometry. Methods Mol Biol 2019, 2030:17-31.

\section{Figures}


a

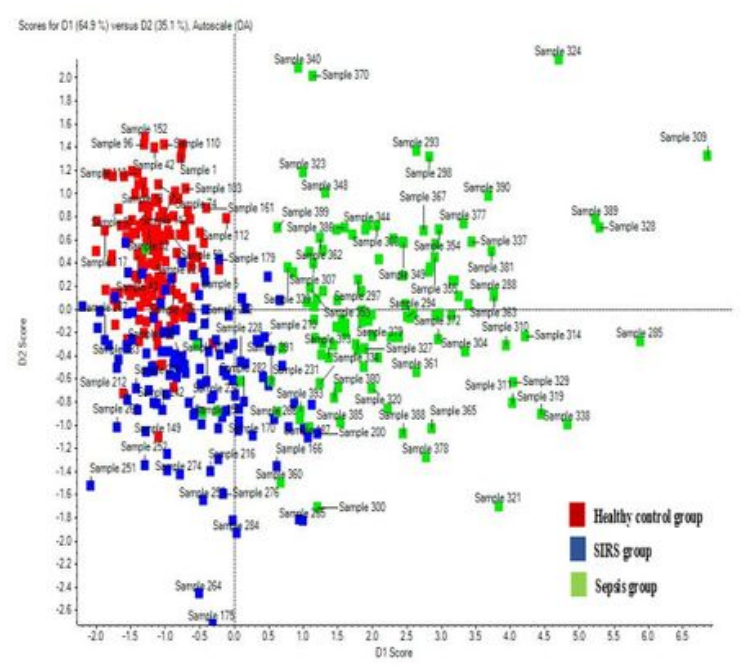

b

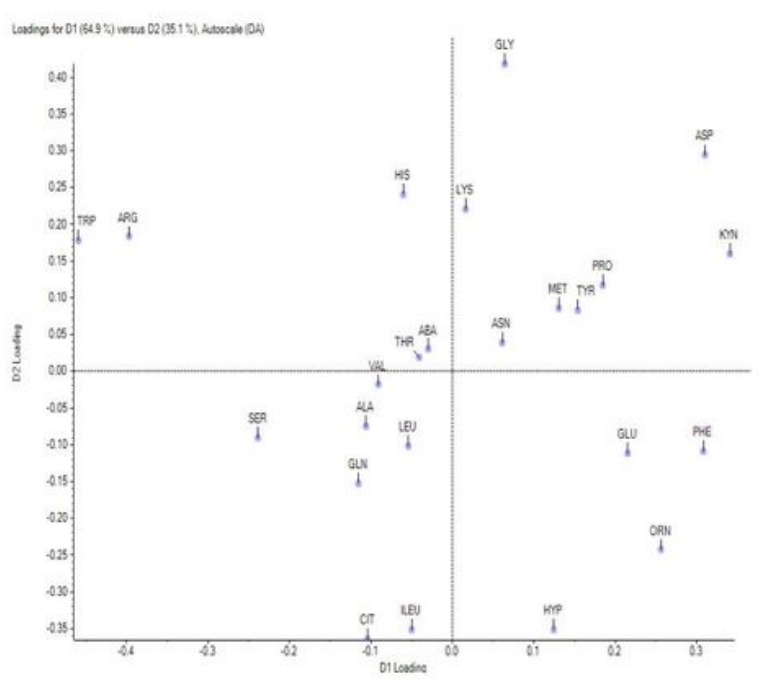

c

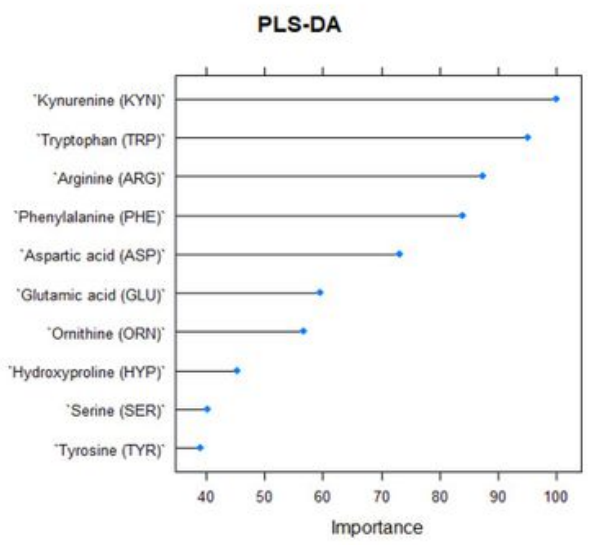

Figure 1

The results of PLS-DA analysis in the training group. a) The score plot of amino acid concentrations. Red, blue, and green squares refer to healthy control, SIRS, and sepsis groups, respectively. b) The loading plot of amino acid concentrations. The blue spots represent amino acids. c) The importance plot. Candidate amino acids are located on the top. 
a

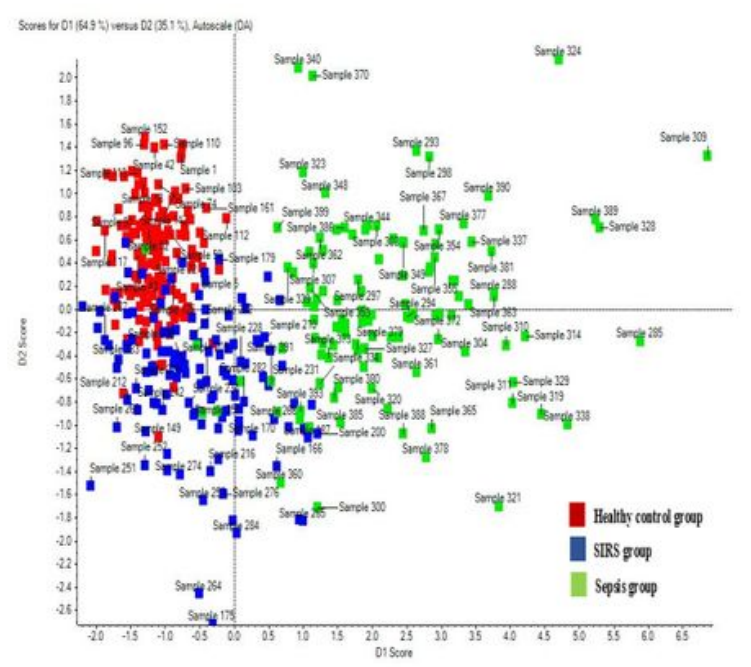

b

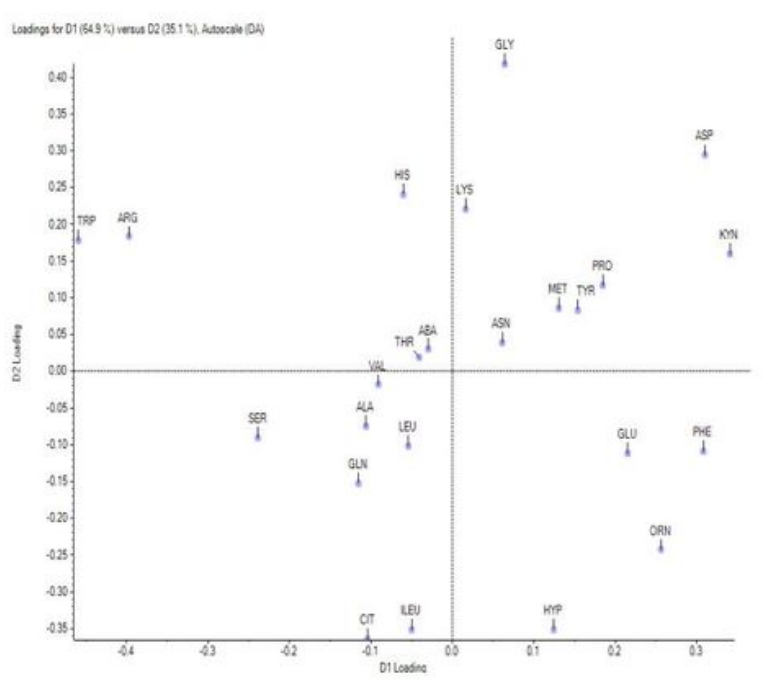

c

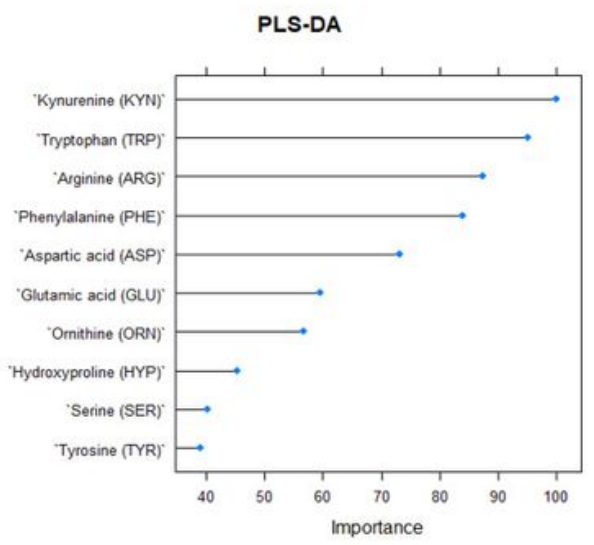

Figure 1

The results of PLS-DA analysis in the training group. a) The score plot of amino acid concentrations. Red, blue, and green squares refer to healthy control, SIRS, and sepsis groups, respectively. b) The loading plot of amino acid concentrations. The blue spots represent amino acids. c) The importance plot. Candidate amino acids are located on the top. 

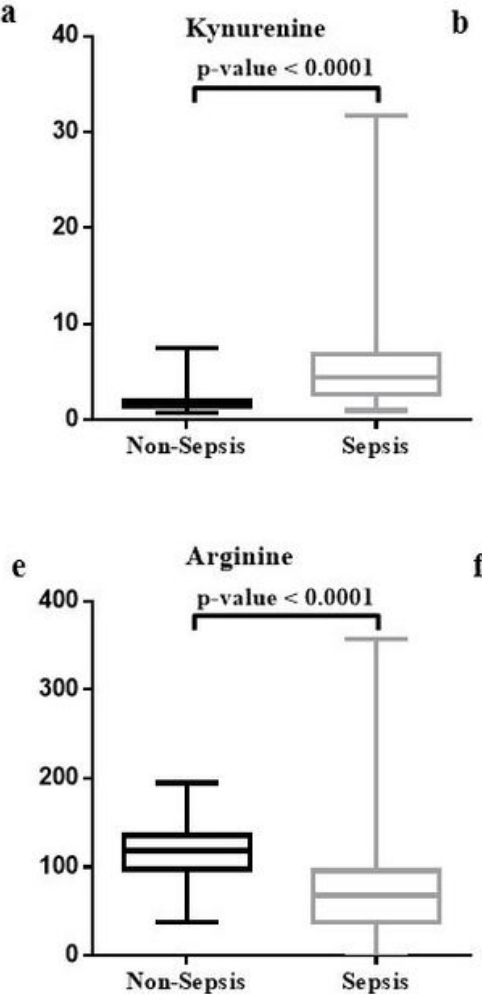

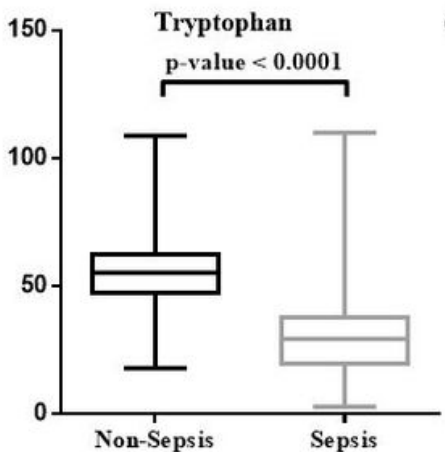

f

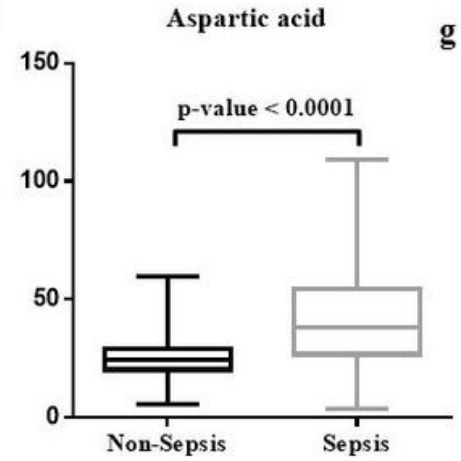

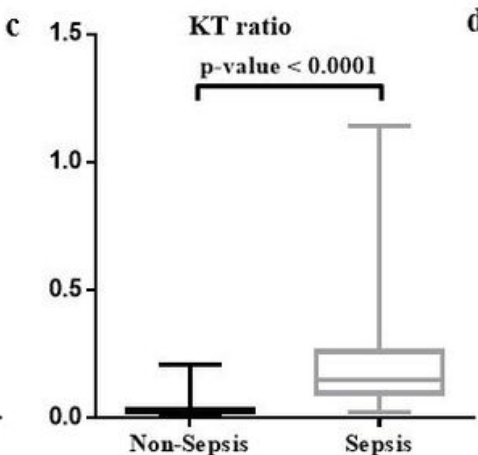

d

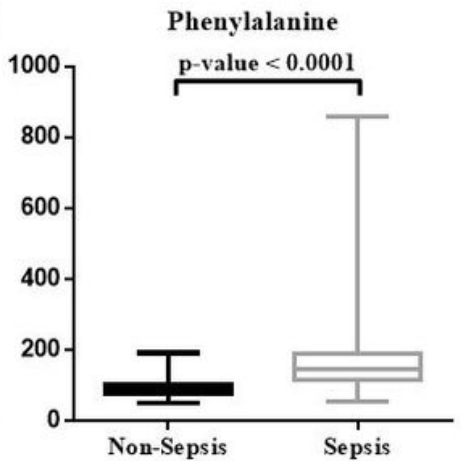

g

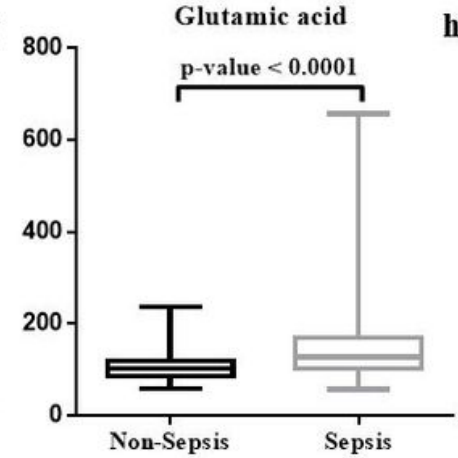

Glutamine

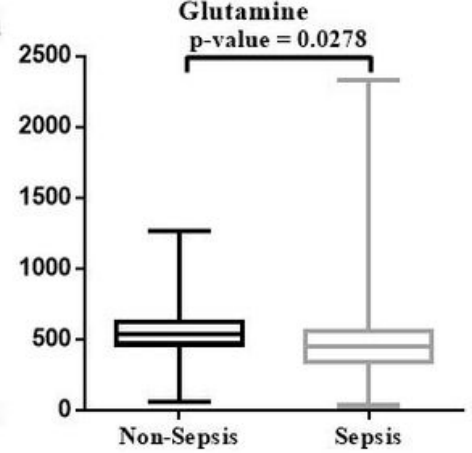

Figure 2

Comparison of candidate amino acid levels using the Mann-Whitney $U$ test in the training group. a) kynurenine, b) tryptophan, c) KT ratio, d) phenylalanine, e) arginine, f) aspartic acid, g) glutamic acid, h) glutamine. The concentrations are expressed in $\mu \mathrm{M}$. Distributions are shown as box-plots where the central line indicates the median concentration. 

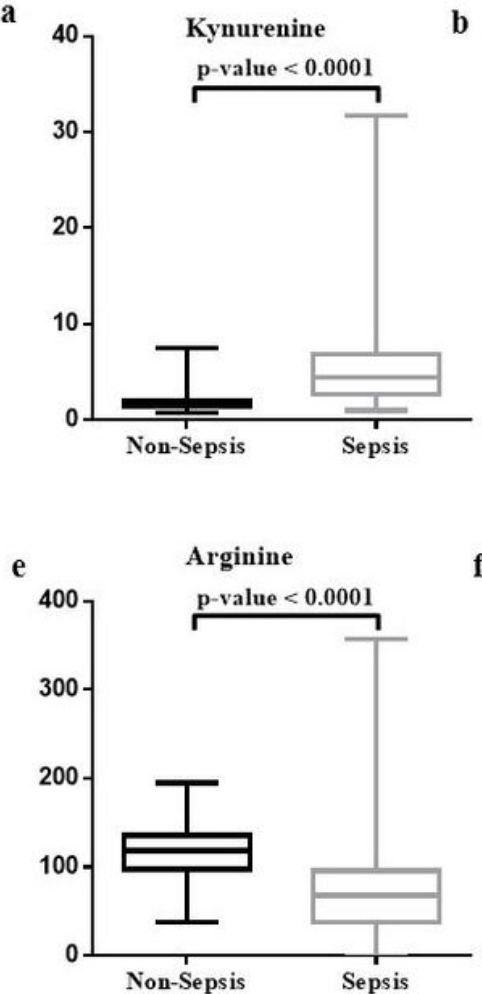

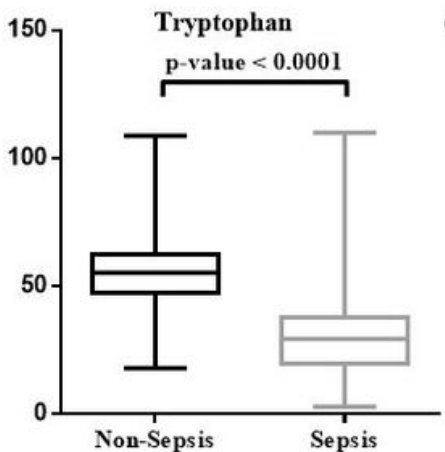

f

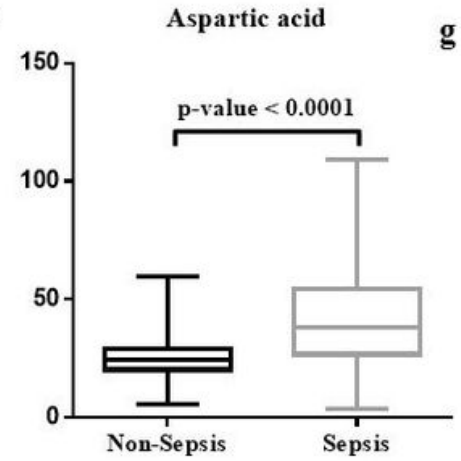

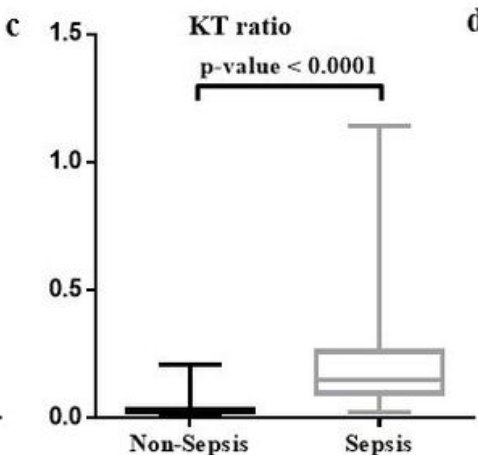

d

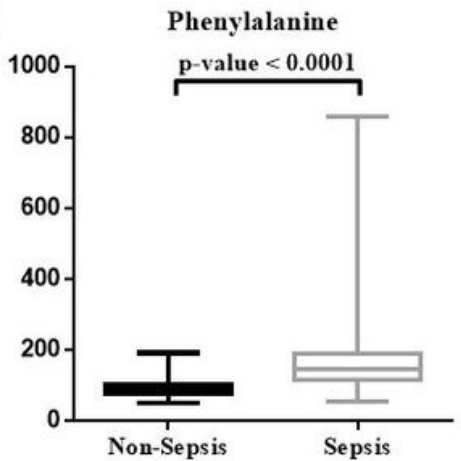

g

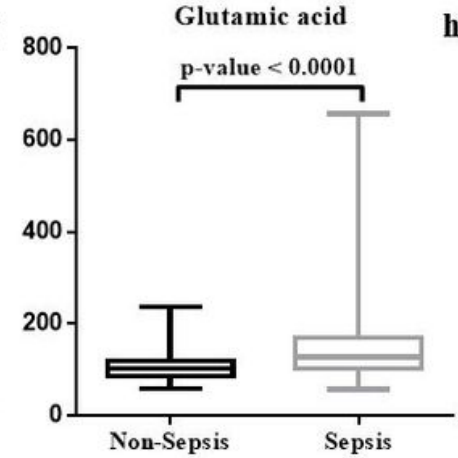

Glutamine

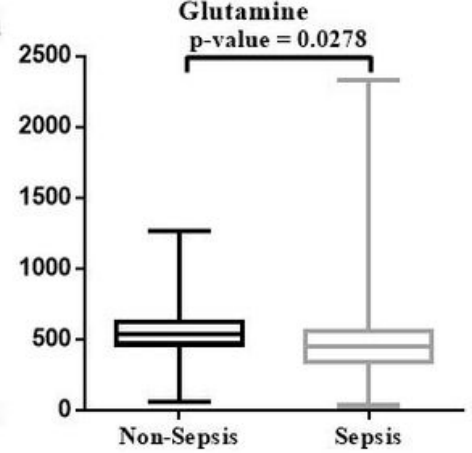

Figure 2

Comparison of candidate amino acid levels using the Mann-Whitney $U$ test in the training group. a) kynurenine, b) tryptophan, c) KT ratio, d) phenylalanine, e) arginine, f) aspartic acid, g) glutamic acid, h) glutamine. The concentrations are expressed in $\mu \mathrm{M}$. Distributions are shown as box-plots where the central line indicates the median concentration. 
a Multivariate Index

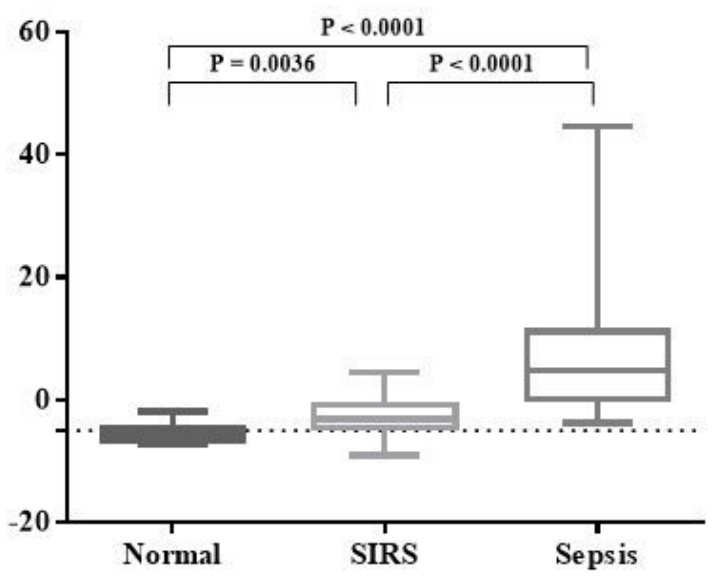

c

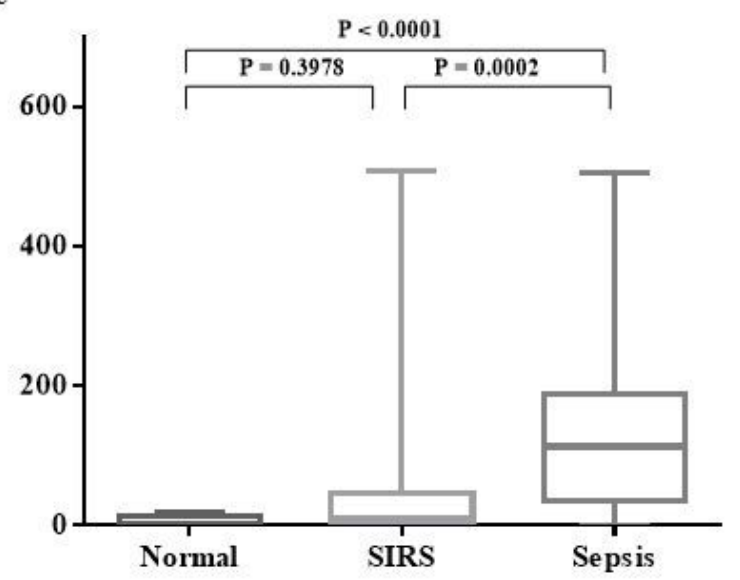

b

Procalcitonin

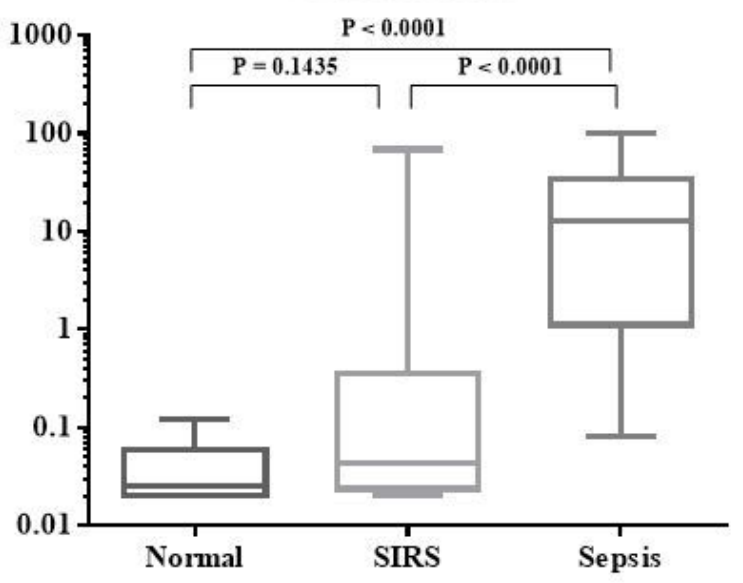

d

WBC

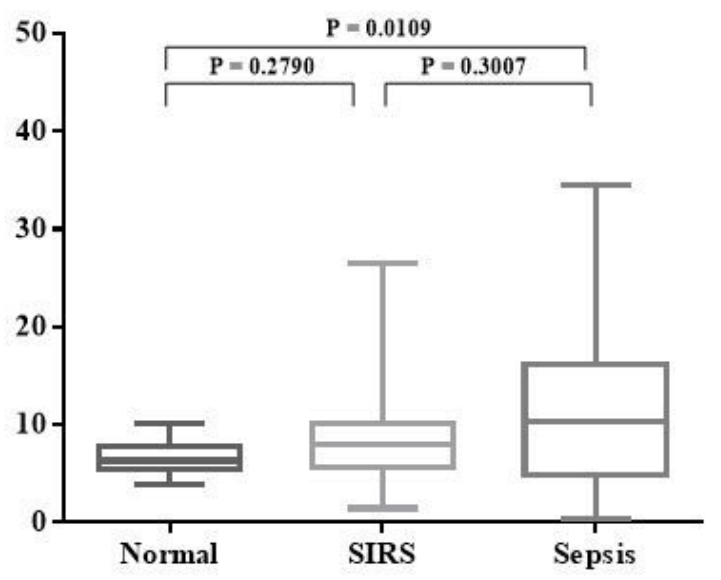

\section{Figure 3}

Results of the Kruskal-Wallis test among the three subgroups (normal, SIRS, sepsis) in the validation group. In all cases, the p-value was $<0.05$. a) developed multivariate index, b) procalcitonin ( $\mathrm{ng} / \mathrm{mL}), \mathrm{c})$ C-reactive protein $(\mathrm{mg} / \mathrm{L})$, d) white blood cell $(103 / \mu \mathrm{L})$. Distributions are shown as box-plots where the central line indicates the median concentration. 
a Multivariate Index

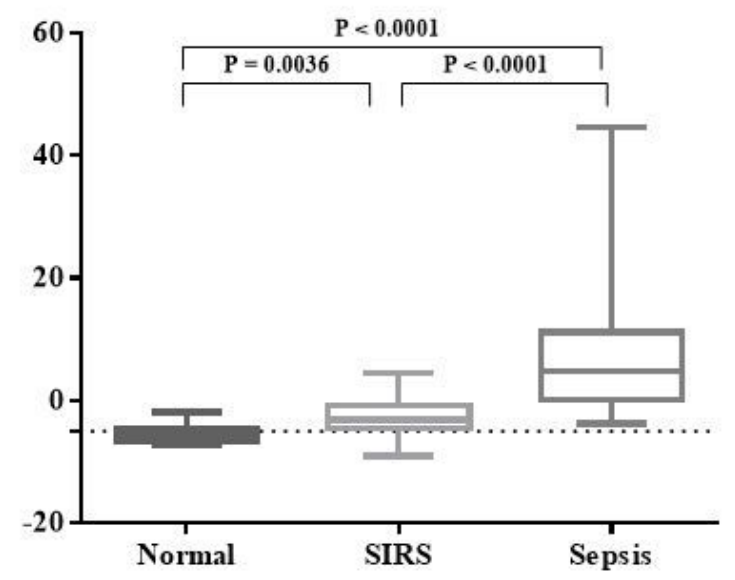

c

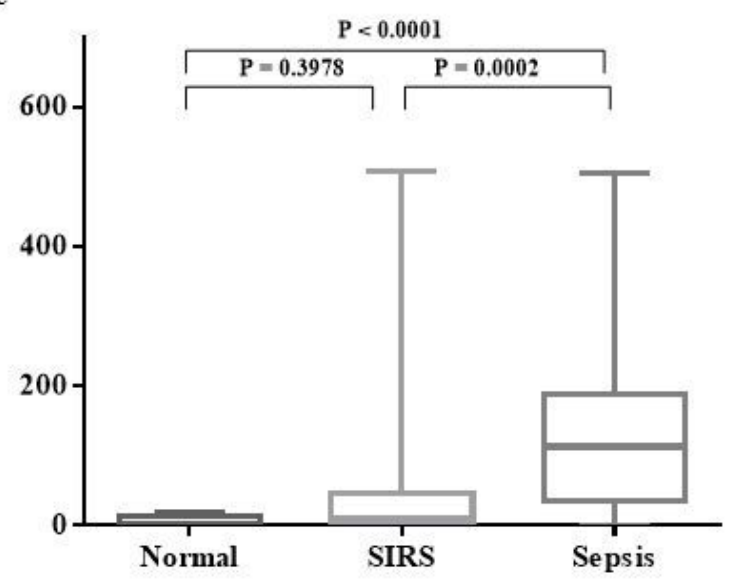

b

Procalcitonin

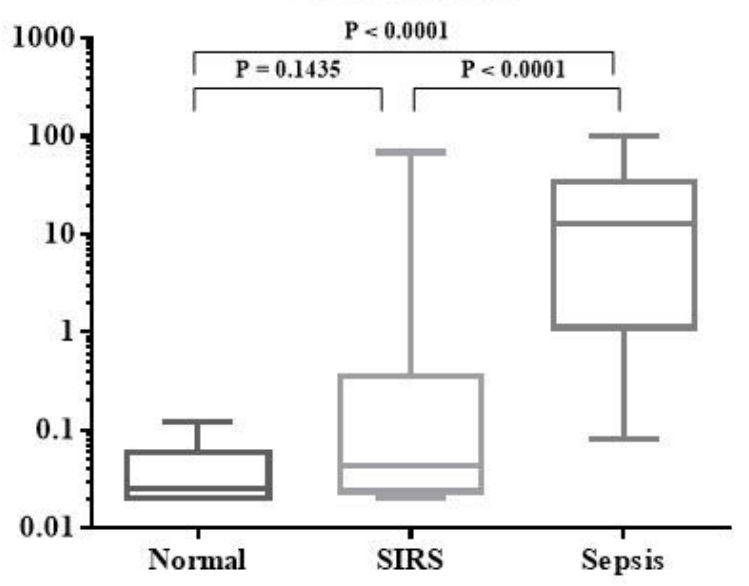

d

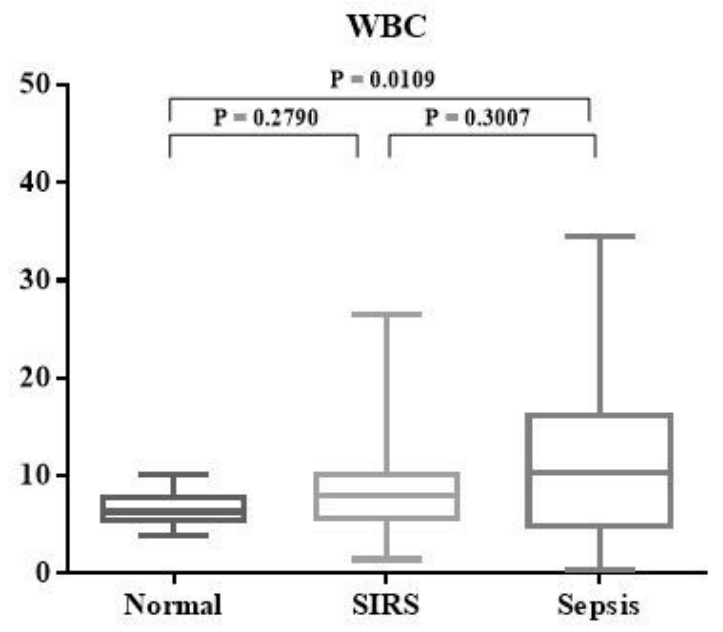

\section{Figure 3}

Results of the Kruskal-Wallis test among the three subgroups (normal, SIRS, sepsis) in the validation group. In all cases, the $\mathrm{p}$-value was $<0.05$. a) developed multivariate index, b) procalcitonin ( $\mathrm{ng} / \mathrm{mL}), \mathrm{c}$ ) C-reactive protein $(\mathrm{mg} / \mathrm{L}), \mathrm{d})$ white blood cell $(103 / \mu \mathrm{L})$. Distributions are shown as box-plots where the central line indicates the median concentration.

\section{Supplementary Files}

This is a list of supplementary files associated with this preprint. Click to download.

- Additionalinformationfile1.docx

- Additionalinformationfile1.docx

- Additionalinformationfile2.docx

- Additionalinformationfile2.docx

- Additionalinformationfile3.docx

- Additionalinformationfile3.docx 
- Additionalinformationfile4.docx

- Additionalinformationfile4.docx

- Additionalinformationfile5.jpg

- Additionalinformationfile5.jpg

- Additionalinformationfile6.JPG

- Additionalinformationfile6.JPG

- Additionalinformationfile7.jpg

- Additionalinformationfile7.jpg

- Additionalinformationfile8.JPG

- Additionalinformationfile8.JPG

- graphicalabstract.JPG

- graphicalabstract.JPG 